\title{
Greens Functions, Hamiltonians and Modular Automorphisms
}

\author{
Ola Bratteli \\ Zentrum für interdisziplinäre Forschung der Universität Bielefeld, D-4800 Bielefeld, \\ Federal Republic of Germany \\ Derek W. Robinson \\ Université d'Aix-Marseille II, Luminy, Marseille, and Centre de Physique Theorique, CNRS, \\ F-13274 Marseille Cedex 2, France
}

\begin{abstract}
We demonstrate, under circumstances that allow the construction of a "thermodynamic" hamiltonian, that Gibbs equilibrium states $\omega$ are modular states in the Tomita-Takesaki sense. The thermodynamic Greens functions $G$ are connected to these modular states, and the associated group of modular automorphisms $\sigma$, by the identification

$$
G(A, B ; t)=\omega\left(A \sigma_{t}(B)\right)
$$

( $A$ and $B$ are observables) whenever the thermodynamic Hamiltonian is selfadjoint and defines a derivation of the algebra of observables in a certain sense. Our results apply to a class of interacting quantum gases at small fugacity and Bose gases with repulsive interactions at all fugacities $z<1$.
\end{abstract}

\section{Introduction}

Although the time-development of thermodynamic systems in quantum statistical mechanics is barely understood, some progress has been made on the questions of existence and characterization of equilibrium. The existence problem has been tackled by establishing that the limits

$$
\tau_{t}(A)=\lim _{\Lambda \rightarrow \infty} e^{i H_{\Lambda} t} A e^{-i H_{\Lambda} t}
$$

of the time development of finite systems $\Lambda$ exist in some suitable sense. $\left(H_{\Lambda}\right.$ represents the Hamiltonian of a finite system $\Lambda, A$ a fixed observable, and the limit indicates the thermodynamic limit of an idealized infinite system.) Two types of limit, i.e. types of convergence, have been distinguished.

In the best cases (free fermi gas, "short range" spin systems) the above limits exist for all $A$ in a $C^{*}$-algebra $\mathfrak{A}$ of quasi-local observables and $t \rightarrow \tau_{t}$ defines a strongly continuous one-parameter group of automorphisms of $\mathfrak{A}$ [1-3]. Under these circumstances one can also show that all Gibbs equilibrium states (limits 
of the type

$$
\omega(A)=\lim _{\Lambda \rightarrow \infty} \operatorname{Tr}\left(e^{-H_{\Lambda}} A\right) / \operatorname{Tr}\left(e^{-H_{\Lambda}}\right), A \in \mathfrak{A},
$$

are modular states in the sense of Tomita-Takesaki [4]. Furthermore the modular automorphism coincides with $\tau$. Expressed in another way, $\omega$ satisfies the $\tau-K M S$ condition.

In other cases (low density Bose and Fermi gases, "long range" spin systems) the limit dynamics has been established for Gibbs states through use of the time dependent Greens functions

$$
G(A, B ; t)=\lim _{\Lambda \rightarrow \infty} \operatorname{Tr}\left(e^{-H_{\Lambda}} A e^{i H_{\Lambda} t} B e^{\left.-i H_{\Lambda} t\right)} / \operatorname{Tr}\left(e^{-H_{\Lambda}}\right)\right.
$$

etc. [5-9]. One would expect the equilibrium characteristics of the latter problems to resemble that of the former. In particular one would reasonably believe that

a) the Gibbs equilibrium states are modular states in the Tomita-Takesaki sense,

b) the modular automorphism and the Greens functions are connected by the relation

$$
G(A, B ; t)=\omega\left(A \sigma_{t}(B)\right) .
$$

In this paper we tackle these two problems. The first is resolved and the second reduced to a question of self-adjointness of the Hamiltonian naturally associated with the infinite system and certain density estimates for this system.

\section{Principal Results}

In this section we discuss certain properties of the states obtained from the thermodynamic limit in quantum statistical mechanics. We work in a semi-abstract setting which is devised to cover various models of statistical mechanics.

The states of a quantum-mechanical system are determined by the states of its finite subsystems and these in turn are defined via a directed set $\mathfrak{H}_{A}$ of Hilbert spaces which are such that if $\Lambda_{1}<\Lambda_{2}$ then $\mathfrak{H}_{\Lambda_{1}}$ is identifiable as a subspace of $\mathfrak{H}_{\Lambda_{2}}$. In applications the indices $\Lambda$ run over the bounded open sets of $\mathbb{R}^{v}$, or the finite subsets of $\mathbb{Z}^{v}$, and the direction is by inclusion.

The observables of the system can be described by a family $\mathfrak{U}_{\Lambda}$ of $C^{*}$-algebras which we will take to form an isotonic family in the following sense.

A family $\mathfrak{C}_{\Lambda}$ of $*$-algebras is defined to be an isotonic family if

1. each $\mathfrak{C}_{A}$ is isomorphic to an irreducible subalgebra of $\mathfrak{Q}\left(\mathfrak{H}_{A}\right)$ containing the identity,

2. if $\Lambda_{1}<\Lambda_{2}$ then

$$
\mathfrak{C}_{\Lambda_{1}} \subset \mathfrak{C}_{\Lambda_{2}} \text {. }
$$

Note that to each isotonic family, for example the $\mathfrak{U}_{\Lambda}$, we may associate a global $C^{*}$-algebra, for example $\mathfrak{U}$, which is defined as the uniform closure of the union of the $\mathfrak{U}_{\Lambda}$. 
In order to define the usual Gibbs states of our system it is necessary to introduce a family of Hamiltonians $H_{\Lambda}$. Each $H_{\Lambda}$ is assumed be a lower semibounded self-adjoint operator, on the corresponding Hilbert space $\mathfrak{H}_{\Lambda}$, with the property that

$$
Z_{\Lambda}(\beta)=\operatorname{Tr}_{\mathfrak{H}_{\Lambda}}\left(e^{-\beta H_{\Lambda}}\right)<+\infty
$$

for all $\beta>0$.

The family of Gibbs states $\omega_{\Lambda}$, associated with a system of the above type, are now introduced by

$$
\omega_{\Lambda}(A)=\operatorname{Tr}_{\mathfrak{H}_{\Lambda}}\left(e^{-\beta H_{\Lambda}} A\right) / \operatorname{Tr}_{\mathfrak{H}_{\Lambda}}\left(e^{-\beta_{\Lambda}}\right)
$$

where $A \in \mathfrak{U}_{\Lambda}$. Thus $\omega_{\Lambda}$ is a state over the $C^{*}$-algebra $\mathfrak{U}_{\Lambda}$. Note that as $\mathfrak{U}_{\Lambda}$ is a subalgebra of $\mathfrak{U}$ the state $\omega_{\Lambda}$ has an extension to a state over $\mathfrak{U}$. Further as $\mathfrak{U}$ contains the identity its state space is weak *-compact. Thus, implicitly identifying the $\omega_{\boldsymbol{A}}$ with one of their extensions to $\mathfrak{U}$, we can assert the existence of weak *-limit points

$$
\omega(A)=\lim _{\alpha} \omega_{\Lambda_{\alpha}}(A)
$$

where $A \in \mathfrak{U}_{\Lambda_{\beta}}$ and $\Lambda_{\beta}<\Lambda_{\alpha}$. The $\omega$ obtained in this manner extend by continuity to give states over $\mathfrak{A}$. We wish to study such limit states and for this it will be necessary to make further assumptions concerning the Hamiltonians $H_{\Lambda}$.

In the following discussion we will encounter commutator expressions of the form $\left[H_{A}, A\right]$ and the additional assumptions we now introduce are designed to ensure that these expressions have a well-defined meaning for a large enough set of $A$. We assume:

H1. there exists an isotonic family of *-algebras $\mathfrak{D}_{\Lambda} \subseteq \mathfrak{U}_{\Lambda}$ such that if $A \in \mathfrak{D}_{\Lambda}$ then $A D\left(H_{A}\right) \subseteq D\left(H_{A}\right)$;

$\mathrm{H} 2$. the operators

$$
\left[H_{\Lambda}, A\right] e^{-\beta H_{A}}
$$

are of trace-class for all $A \in \mathfrak{D}_{\Lambda}$ and all $\beta>0$;

H3. the Gibbs states $\omega_{\Lambda}$ are such that

$$
\omega_{\Lambda}\left(\left[H_{A}, A\right]^{*}\left[H_{\Lambda}, A\right]\right)<c_{A}
$$

where $c_{A}$ is independent of $\Lambda$ and the bound is valid for all $A \in \mathfrak{D}_{\Lambda}$ and all $\Lambda$.

Note that condition 1 implies the operator of condition 2 is well defined. In condition 3 the definition of $\omega_{A}$ is extended to unbounded operators for which the appropriate operators are of trace-class.

Our first result is the following

Theorem 1. Let $\left\{\omega_{A}\right\}$ denote the Gibbs states associated with the family of Hamiltonians $\left\{H_{A}\right\}$ and let $\omega$ denote a limit point of the $\omega_{A}$ in the weak ${ }^{*}$ topology. Let the $\left\{H_{A}\right\}$ satisfy condition $H 1-H 3$ above. 
Next assume there exists an isotonic family $\mathfrak{B}_{\Lambda}$ of ${ }^{*}$-algebras such that $\mathfrak{B}_{\Lambda} \subset \mathscr{D}_{\Lambda}$ and the algebra $\mathfrak{B}$ formed by the union of the $\mathfrak{B}_{\Lambda}$ has the following properties

1. $\mathfrak{B}$ is separable in the uniform topology,

2. the restriction to $\mathfrak{B}$ of the representation $\pi_{\omega}$ generated by $\omega$ is weakly dense in $\pi_{\omega}(\mathfrak{Q})^{\prime \prime}$.

It follows that $\omega$ is a modular state over $\mathfrak{A}$, i.e. the vector $\Omega_{\omega}$ canonically associated with $\omega$ and $\pi_{\omega}$ which is cyclic for $\pi_{\omega}$ is also separating for $\pi_{\omega}^{\prime \prime}$.

Remark. The introduction of $\mathfrak{B}$ appears somewhat artificial and in applications in redundant whenever the algebra $\mathfrak{U}$ is itself norm-separable. The suppositions are devised to cover the case of the Bose gas where $\mathfrak{A}$ is non-separable.

M. Winnink has kindly informed us that he had previously proved a similar theorem (see [7]). He assumed continuity of the limit Greens functions in time instead of our assumptions H1-H3. Thus Theorem 1 follows from his result and our Lemma 1. For completeness we include our proof.

The proof of the theorem uses the (one-time) Greens functions $G_{A}$ which are defined by

$$
G_{\Lambda}(A, B ; t)=\operatorname{Tr}_{\mathfrak{G}_{\Lambda}}\left(e^{-(\beta+i t) H_{\Lambda}} A e^{i t H_{\Lambda}} B\right) / Z_{\Lambda}(\beta)
$$

where $A, B \in \mathfrak{U}_{A}$ and $t \in \mathbb{R}$. We begin by listing a number of properties of these functions which will be needed in the sequel.

1. $\left|G_{\Lambda}(A, B ; t)\right|^{2} \leqq \omega_{\Lambda}\left(A A^{*}\right) \omega_{\Lambda}\left(B^{*} B\right), A, B \in \mathfrak{U}_{\Lambda}$,

2. $\sum_{i j} \overline{c_{i}} c_{j} G_{\Lambda}\left(A^{*}, A ; t_{i}-t_{j}\right) \geqq 0$

for all $c_{i}, c_{j} \in \mathbb{C}$ and $A \in \mathfrak{A}_{A}$.

3. If $B \in \mathfrak{D}_{\Lambda}$ then $G_{\Lambda}(A, B ; t)$ is once differentiable and

$$
\left|\frac{\partial}{\partial t} G_{\Lambda}(A, B ; t)\right|^{2} \leqq \omega_{\Lambda}\left(A A^{*}\right) \omega_{\Lambda}\left(\left[H_{\Lambda}, B\right]^{*}\left[H_{\Lambda} B\right]\right) \text {. }
$$

4. If $A, B \in \mathfrak{D}_{\Lambda}$ then $G(A, B ; t)$ is twice differentiable and

$$
\left|\frac{\partial^{2}}{\partial t^{2}} G_{\Lambda}(A, B ; t)\right|^{2} \leqq \omega_{\Lambda}\left(\left[H_{\Lambda}, A\right]\left[H_{\Lambda}, A\right]^{*}\right) \omega_{\Lambda}\left(\left[H_{\Lambda}, B\right]^{*}\left[H_{\Lambda}, B\right]\right) \text {. }
$$

5. If $f$ is a function whose Fourier transform $\tilde{f}$ is in the class $\mathfrak{D}(\mathbb{R})$ of Schwartz then

$$
\int d t f(t) G_{\Lambda}(A, B ; t)=\int d t f(t+i \beta) G_{\Lambda}(B, A ;-t)
$$

for all $A, B \in \mathfrak{U}_{\Lambda}$.

The last condition is a rephrasing, in the form of [10], of the Kubo-MartinSchwinger (KMS) boundary condition. This condition expresses that the $G_{A}$ have analytic extensions in the strip $0<\operatorname{Im} z<\beta$ which are continuous and bounded on the closure of the strip. The boundary values satisfy

$$
G_{\Lambda}(A, B ; t+i)=G_{\Lambda}(B, A ;-t)
$$

for all $A, B \in \mathfrak{A}_{A}$ and $t \in \mathbb{R}$. 

that

We next consider limits of these Greens functions. We assume throughout

$$
\omega(A)=\lim _{\alpha} \omega_{\Lambda_{\alpha}}(A), A \in \cup_{\Lambda} \mathfrak{A}_{\Lambda}
$$

exists for some net of $\Lambda_{\alpha}$ and then consider limits of subsequences of the Greens functions.

Lemma 1. Adopt the assumptions of Theorem 1. For all $A, B \in \mathfrak{B}$ and $t \in \mathbb{R}$ there exists a subsequence $\Lambda_{n}$ such that the Greens functions

$$
G(A, B ; t)=\lim _{n \rightarrow \infty} G_{A_{n}}(A, B ; t)
$$

exist. These functions are once-differentiable and

$$
\frac{\partial}{\partial t} G(A, B ; t)=\lim _{n \rightarrow \infty} \frac{\partial}{\partial t} G_{\Lambda_{n}}(A, B ; t) .
$$

Proof. The existence of the $G(A, B ; t)$ follows from the work of Ruskai [5]. Ruskai's application of the Arzela-Ascoli theorem is valid in the present context because of the assumed separability of $\mathfrak{B}$ and property $\mathrm{H} 3$.

Next note that

$$
\left|\frac{\partial^{2}}{\partial t^{2}} G_{A}(A, B ; t)\right|^{2}<c_{A} c_{B}
$$

uniformly in $\Lambda$ and hence by a simple application of Taylor's series

$$
\begin{aligned}
& \left|\frac{\partial}{\partial t} G_{\Lambda_{n}}(A, B ; t)-\frac{\partial}{\partial t} G_{A_{m}}(A, B ; t)\right| \\
& \leqq \\
& \quad\left|G_{\Lambda_{n}}(A, B ; t+h)-G_{A_{n}}(A, B ; t+h)\right| /|h| \\
& \quad+\left|G_{A_{n}}(A, B ; t)-G_{A_{m}}(A, B ; t)\right| /|h|+\left(C_{A} C_{B}\right)^{1 / 2}|h| .
\end{aligned}
$$

Hence the limit of the derivatives of the $G_{A_{n}}$ exist. But another application of Taylor's series gives

$$
\left|G_{A_{n}}(A, B ; t+h)-G_{A_{n}}(A, B ; t)-h \frac{\partial}{\partial t} G_{A_{n}}(A, B ; t)\right| \leqq 2^{-1} h^{2}\left(C_{A} C_{B}\right)^{1 / 2} .
$$

The described result follows immediately.

Next we consider the properties of $G$ considered as a sesquilinear form over the representation space $\mathfrak{H}_{\omega}$ associated with the pair $(\omega, \mathfrak{U})$

Lemma 2. Adopt the assumptions of Theorem 1 and let $G$ denote the Greens functions defined in Lemma 1.

There exists a Hilbert space $\mathfrak{R}_{\omega} \supset \mathfrak{H}_{\omega}$ and a strongly continuous one-parameter family of unitary operators $V_{t}$ on $\mathfrak{\Re}_{\omega}$ such that $\mathfrak{\Re}_{\omega}=\bigvee_{t} V_{t} \mathfrak{H}_{\omega}$ and

$$
G(A, B ; t)=\left(\Omega_{\omega}, \pi_{\omega}(A) V_{t} \pi_{\omega}(B) \Omega_{\omega}\right) .
$$


Further if $f$ is a function such that $\tilde{f} \in \mathfrak{D}$ then

$$
\begin{aligned}
& \int d t f(t)\left(\Omega_{\omega}, \pi_{\omega}(A) V_{t} \pi_{\omega}(B) \Omega_{\omega}\right) \\
& =\int d t f(t+i \beta)\left(\Omega_{\omega}, \pi_{\omega}(B) V_{-t} \pi_{\omega}(A) \Omega_{\omega}\right)
\end{aligned}
$$

for all $A, B \in \mathfrak{B}$.

Proof. The first part of the lemma may be deduced from Ruskai's discussion [5] of multi-time Greens functions or may be deduced directly by the following argument:

From property 1 of $G_{A}$ it follows that

$$
\begin{aligned}
|G(A, B, t)|^{2} & \leqq \omega\left(A A^{*}\right) \omega\left(B^{*} B\right) \\
& =\left\|\pi_{\omega}\left(A^{*}\right) \Omega_{\omega}\right\|^{2}\left\|\pi_{\omega}(B) \Omega_{\omega}\right\|^{2} .
\end{aligned}
$$

Hence the function $G$ defines a one-parameter family of bounded sesquilinear forms on $\pi_{\omega}(\mathfrak{B}) \Omega_{\omega} \times \pi_{\omega}(\mathfrak{B}) \Omega_{\omega}$. The closures of these forms define a one-parameter family $t \in \mathbb{R} \rightarrow X_{t} \in \mathfrak{L}\left(\mathfrak{H}_{\omega}\right)$ of bounded operators on $\mathfrak{H}_{\omega}$ with bound

$$
\left\|X_{t}\right\| \leqq 1
$$

By Lemma 1, this family is weakly continuous. From property 2 of $G_{A}$ it follows that $t \rightarrow G\left(A^{*}, A, t\right)$ is positive definite in time, hence

$$
\begin{aligned}
& X_{0}=\mathbb{1}, \quad X_{t}^{*}=X_{-t} \\
& \sum_{i j} c_{i}^{-} c_{j} X_{t_{t}-t_{j}} \geqq 0, \quad c_{i} \in \mathbb{C} .
\end{aligned}
$$

Now one may apply the Nagy extension theorem, [11], page 452, to deduce the existence of $\mathfrak{R}_{\omega}$ and $V$. If $P$ denotes the orthogonal projector, on $\mathfrak{R}_{\omega}$, with range $\mathfrak{H}_{\omega}$ one has $X_{t}=P V_{t} P$.

The second statement of the lemma follows from Property 5 of $G_{A}$ and Lebesques convergence theorem.

Proof of Theorem 1. If $A \in \pi_{\omega}^{\prime \prime}$, then it follows from the strong*-density of $\pi_{\omega}(\mathfrak{B})$ in $\pi_{\omega}(\mathfrak{A})^{\prime \prime}$ that one may choose a sequence $A_{n} \in \mathfrak{B}$ such that

$$
\lim _{n} \pi_{\omega}\left(A_{n}\right) \Omega_{\omega}=A \Omega_{\omega}, \quad \lim _{n} \pi_{\omega}\left(A_{n}^{*}\right) \Omega_{\omega}=A^{*} \Omega_{\omega} .
$$

Thus from Lemma 2 one has

$$
\int d t f(t)\left(A \Omega_{\omega}, V_{t} A \Omega_{\omega}\right)=\int d t f(t+i \beta)\left(A^{*} \Omega_{\omega}, V_{-t} A^{*} \Omega_{\omega}\right)
$$

for all $A \in \pi_{\omega}^{\prime \prime}$ and $f$ with $\tilde{f} \in \mathcal{D}$. Now suppose $A \in \pi_{\omega}^{\prime \prime}$ is such that $A^{*} \Omega_{\omega}=0$. One concludes that

$$
\int d t f(t)\left(A \Omega_{\omega}, V_{t} A \Omega_{\omega}\right)=0 .
$$

But as $V$ is a strongly continuous unitary group it easily follows that $A \Omega_{\omega}=0$.

Finally choosing $A$ as above and taking any $B \in \pi_{\omega}^{\prime \prime}$ one is forced to the conclusion

$$
(A B)^{*} \Omega_{\omega}=B^{*}\left(A^{*} \Omega_{\omega}\right)=0
$$


and hence, by the foregoing argument, applied to $A B$, instead of $A$

$$
A B \Omega_{\omega}=0 \text {. }
$$

But $\Omega_{\omega}$ is cyclic for $\pi_{\omega}$ in $\mathfrak{H}_{\omega}$ and hence $A=0$, i.e. $\Omega_{\omega}$ is separating for $\pi_{\omega}^{\prime \prime}$. This completes the proof of the theorem.

Next consider the operators $X$ associated with $G$ and their unitary extensions $V$. If $K$ is the infinitesimal generator of $V$, on $\mathfrak{K}_{\omega}$, and $E_{K}$ its spectral family then the KMS condition of Lemma 2 states that when $A \in \pi_{\omega}^{\prime \prime}$ then

$$
\int d\left(A \Omega_{\omega}, E_{K}(\lambda) A \Omega_{\omega}\right) \tilde{f}(\lambda)=\int d\left(A^{*} \Omega_{\omega}, E_{K}(\lambda) A^{*} \Omega_{\omega}\right) \tilde{f}(\lambda) e^{-\beta \lambda} .
$$

One easily concludes that

$$
\begin{aligned}
\left\|A \Omega_{\omega}\right\|^{2} & =\left(A \Omega_{\omega}, A \Omega_{\omega}\right)=\int d\left(A^{*} \Omega_{\omega}, E(\lambda) A^{*} \Omega_{\omega}\right) e^{-\beta \lambda} \\
& =\left\|e^{-\beta K / 2} A^{*} \Omega_{\omega}\right\| .
\end{aligned}
$$

In particular $\pi_{\omega}^{\prime \prime} \Omega_{\omega} \in D(\exp \{-\beta K / 2\})$. Further, as $\Omega_{\omega}$ is cyclic and separating for $\pi_{\omega}^{\prime \prime}$, there exists, by the modular theory of Tomita-Takesaki, a group of automorphisms of $\pi_{\omega}^{\prime \prime}$, implemented by unitaries $\Delta_{\omega}^{i t}$, such that the self-adjoint operator $\Delta_{\omega}$ satisfies

$$
\left\|A \Omega_{\omega}\right\|^{2}=\left\|\Delta_{\omega}^{\frac{1}{2} \beta} A^{*} \Omega_{\omega}\right\| .
$$

Further $\pi_{\omega}^{\prime \prime} \Omega_{\omega}$ is a core for $\Delta_{\omega}^{\beta}$. Therefore one has

$$
\Delta_{\omega}^{\beta}=P e^{-\beta K} P
$$

where $P$ is the projector on $\mathfrak{R}_{\omega}$ with range $\mathfrak{H}_{\omega}$. Thus if $\mathfrak{K}_{\omega}=\mathfrak{H}_{\omega}$ one has

$$
\Delta_{\omega}^{\beta}=e^{-\beta K}
$$

and

$$
\Delta_{\omega}^{i t \beta}=U_{\beta t} .
$$

In particular

$$
V_{t} \pi_{\omega}^{\prime \prime} V_{-t}=\pi_{\omega}^{\prime \prime}, \quad t \in \mathbb{R}
$$

i.e. if the Greens functions $G$ determine a one-parameter family of unitaries on $\mathfrak{H}_{\omega}$ then these unitaries implement a group of automorphisms of $\pi_{\omega}^{\prime \prime}$ and this group is the modular group of the state $\omega$. Thus it is of interest to find criteria which ensure that the $G$ determine unitaries.

One simple criterion can be given in terms of the Greens functions $G$ and the modular functions $F_{\omega}$ associated with $\omega$. This latter function, defined by

$$
F_{\omega}(A, B ; t)=\left(\Omega_{\omega}, A \Delta_{\omega}^{i t} B \Omega_{\omega}\right)
$$

for $A, B \in \pi_{\omega}^{\prime \prime}$, has an analytic continuation in the strip $0 \leqq \operatorname{Im} z \leqq \beta$. We have concluded above that

$$
F_{\omega}\left(A^{*}, A ; i \beta\right)=G\left(A^{*}, A ; i \beta\right), \quad A \in \pi_{\omega}^{\prime \prime} .
$$

But it also follows that

$$
F_{\omega}\left(A^{*}, A ; i \beta / 2\right) \geqq G\left(A^{*}, A ; i \beta / 2\right) .
$$


This inequality follows by noting that

$$
\begin{aligned}
\Delta_{\omega}^{\beta} & =P e^{-\beta K} P \\
& =\left(P e^{-\beta K / 2} P\right)^{2}+P e^{-\beta K / 2}(I-P) e^{-\beta K / 2} P \\
& \geqq\left(P e^{-\beta K / 2} P\right)^{2} .
\end{aligned}
$$

Hence

$$
\Delta_{\omega}^{\beta / 2} \geqq P e^{-\beta K / 2} P .
$$

Taking matrix element yields the desired result.

Now we may conclude that $G$ determines a one-parameter family of unitary operators, if, and only if,

$$
F_{\omega}\left(A^{*}, A ; i \beta / 2\right)=G\left(A^{*}, A ; i \beta / 2\right)
$$

for all $A \in \pi_{\omega}^{\prime \prime}$. This result follows because the above inequality becomes an equality if, and only if,

$$
P e^{-\beta K / 2}(I-P) e^{-\beta K / 2} P=0
$$

which in turn implies $P=1$, or $\mathfrak{H}_{\omega}=\mathfrak{S}_{\omega}$.

The foregoing criterion does not appear to be useful in applications but it has some interest because $F_{\omega}$ at the point $i \beta / 2$ corresponds to the Wigner-Yanase entropy [12] of the state $\omega$ (or at least to a natural generalization of this concept). The $G$ corresponds to the limit of the entropy of the subsystems and the above criterion is a maximum entropy principle.

Next we turn to a criterion which involves the self-adjointness of a Hamiltonian operator which can be associated with the limit systems.

We have demonstrated in Lemma 1 that, under the assumptions of Theorem 1 , the limit

$$
\frac{\partial}{\partial t} G(A, B ; t)=\lim _{n} \frac{\partial}{\partial t} G_{\Lambda_{n}}(A, B ; t)
$$

exists. But this derivative of $G$ defines a sesquilinear form over the representation space $\mathfrak{H}_{\omega}$ and one has from the properties of $G_{A}$ previously listed

$$
\left|\frac{\partial}{\partial t} G(A, B ; t)\right|^{2} \leqq \omega\left(A A^{*}\right) c_{B} .
$$

We next prove that the sesquilinear form given by $t=0$ defines a symmetric operator on $\mathfrak{H}_{\omega}$, which can be interpreted as the Hamiltonian of the infinite system.

Proposition 1. Adopt the assumptions and notation of Theorem 1. Let $\Lambda_{n}$ be a sequence such that the limits

$$
G(A, B ; t)=\lim _{n \rightarrow \infty} G_{A_{n}}(A, B ; t)
$$

exist for all $A, B \in \mathfrak{B}$ and $t \in \mathbb{R}$ (cf. Lemma 1$)$. 
The derivatives of $G$ determine a symmetric operator $H_{\omega}$ on $\mathfrak{H}_{\omega}$ such that $D\left(H_{\omega}\right)=$ $\pi_{\omega}(\mathfrak{B}) \Omega_{\omega}$ and

$$
i\left(\pi_{\omega}\left(A^{*}\right) \Omega_{\omega}, H_{\omega} \pi_{\omega}(B) \Omega_{\omega}\right)=\left.\frac{\partial}{\partial t} G(A, B ; t)\right|_{t=0}
$$

for $A, B \in \mathfrak{B}$.

Proof. We have noted above that

$$
-i \frac{\partial}{\partial t} G(A, B ; t)_{t=0}=h\left(\pi_{\omega}\left(A^{*}\right) \Omega, \pi_{\omega}(B) \Omega_{\omega}\right)
$$

defines a sesquilinear form $h$ on $\pi_{\omega}(\mathfrak{B}) \Omega_{\omega} \times \pi_{\omega}(\mathfrak{B}) \Omega_{\omega}$ with the continuity property

$$
\left|h\left(\pi_{\omega}\left(A^{*}\right) \Omega_{\omega}, \pi_{\omega}(B) \Omega_{\omega}\right)\right| \leqq\left\|\pi_{\omega}\left(A^{*}\right) \Omega_{\omega}\right\| c_{B}^{1 / 2} .
$$

Thus by the Riesz representation theorem there exists for each $B \in \mathfrak{B}$, a vector $\psi_{B} \in \mathfrak{H}_{\omega}$ such that

$$
h\left(\pi_{\omega}\left(A^{*}\right) \Omega_{\omega}, \pi_{\omega}(B) \Omega_{\omega}\right)=\left(\pi_{\omega}\left(A^{*}\right) \Omega_{\omega}, \psi_{B}\right)
$$

for all $A \in \mathfrak{B}$. Define $H_{\omega}$ by

$$
H_{\omega} \pi_{\omega}(B) \Omega_{\omega}=\psi_{B}, \quad B \in \mathfrak{B} .
$$

In order that this definition determines a bona-fide operator we must show that

$$
\pi_{\omega}(B) \Omega_{\omega}=0 \text { implies } \psi_{B}=0 .
$$

But as $\Omega_{\omega}$ is separating

$$
\pi_{\omega}(B) \Omega_{\omega}=0 \quad \text { implies } \quad B=0
$$

and hence implies $\partial G(A, B ; t) / \partial t=0$. The desired conclusion follows immediately. The symmetry of $H_{\omega}$ results from the easily checked symmetry of $h$.

In the remainder of this section we study the situation that $H_{\omega}$ is essentially self-adjoint on $\pi_{\omega}(\mathfrak{B}) \Omega_{\omega}$. We establish properties of the commutators $\left[H_{A}, B\right]$, $B \in \mathfrak{B}$, and the states $\omega_{\Lambda}, \omega$, which ensure that the modular automorphism group associated with $\omega$ is given by

$$
\sigma_{t}(A)=e^{i t \bar{H}_{\omega}} A e^{-i t \bar{H}_{\omega}}, \quad A \in \pi_{\omega}^{\prime \prime}, t \in \mathbb{R},
$$

where $\bar{H}_{\omega}$ is the self-adjoint closure of $H_{\omega}$.

The basic idea is to establish conditions under which the Heisenberg equations of notion

$$
\frac{d}{d t} e^{i H_{\Lambda} t} B e^{-i H_{\Lambda} t}=e^{i H_{\Lambda} t} i\left[H_{\Lambda}, B\right] e^{-i H_{\Lambda} t}
$$

have an analogue in the equilibrium representation $\pi_{\omega}$. For this one needs the existence of limits of the differentials $i\left[H_{\Lambda}, B\right]$ as $\Lambda$ tends to infinity. In the simplest models, quantum spin systems, these commutators are bounded and converge within the $C^{*}$-algebra $\mathfrak{U}$ even with the long range interactions considered by Ruskai [5]. For continuous systems the situation is more complicated. The 
easiest behaviour occurs when the inter-particle interaction is of finite range and can be characterized by the following subsidiary condition

H4. If $B \in \mathfrak{B}$ there exists a $\Lambda_{B}$ such that

$$
\delta(B)=i\left[H_{A}, B\right]
$$

is independent of $\Lambda$ for $\Lambda \supset \Lambda_{B}$. [ $\delta(B)$ is an unbounded operator affiliated with $\mathfrak{U}_{\Lambda}$ on $\left.\mathfrak{H}_{\Lambda} \cdot\right]$

We also need a method of estimating the magnitudes of the derivatives $\delta(B)$ in the state $\omega$. This is achieved by the following "a priori" density assumptions.

Recall that $\omega$ was defined as a state over the $C^{*}$-algebra $\mathfrak{A}$ by a weak* limit

$\omega(A)=\lim _{\alpha} \omega_{\Lambda_{\alpha}}(A)$

over a net $\Lambda_{\alpha}$. Our first assumption concerns this net.

N1. $\omega$ is a locally normal state over $\mathfrak{A}=\overline{\left(\cup_{\Lambda} \mathfrak{A}_{\Lambda}\right)}$; i.e. for each $\mathfrak{A}_{\Lambda}$ there exists a density matrix $\varrho_{A}$ on $\mathfrak{H}_{A}$ such that

$$
\omega(A)=\operatorname{Tr}_{\mathfrak{H}_{\Lambda}}\left(\varrho_{\Lambda} A\right), \quad A \in \mathfrak{U}_{\Lambda} .
$$

Further the net $\omega_{\Lambda_{\alpha}}$ converges weak ${ }^{*}$ on $\mathfrak{L}=\bigcup_{\Lambda} \mathfrak{L}\left(\mathfrak{S}_{\Lambda}\right)$ to the state $\hat{\omega}$ which is the unique normal extensions of $\omega$ to $\mathfrak{R}$, i.e.

$$
\hat{\omega}(A)=\operatorname{Tr}_{\mathfrak{H}_{\Lambda}}\left(\varrho_{\Lambda} A\right), A \in \mathfrak{Q}\left(\mathfrak{H}_{\Lambda}\right) .
$$

In applications we will demonstrate that this rather unnatural assumption follows from estimates on the particle energy density. Our next assumptions can be interpreted as estimates on the particle density.

$\mathrm{N} 2{ }^{1}$. There exist positive operators $N_{\Lambda}$ affiliated with $\mathfrak{S}\left(\mathfrak{H}_{\Lambda}\right)$ and constants $c_{m}(\Lambda)$, such that

$$
\omega_{\Lambda^{\prime}}\left(N_{\Lambda}^{m}\right) \leqq c_{m}(\Lambda) \quad m=1,2, \ldots
$$

for all $\Lambda^{\prime} \supset \Lambda$.

$\mathrm{N} 3^{1}$. For $B \in \mathfrak{B}$ there exists an $m$ such that

$$
\delta(B)\left(N_{\Lambda}^{m}+1\right)^{-1}
$$

is bounded for some $\Lambda \supset \Lambda_{B}$.

Remark 1. If $N$ is an unbounded positive operator affiliated with a von Neumann algebra $\mathfrak{M}$ and $\omega$ is a normal state on $\mathfrak{M}$ then $\omega(N)$ has a canonical definition (see for example, [13] Section 3). If N1 and N2 are satisfied then [13] Corollary 3.3 establishes that

$$
\omega\left(N_{\Lambda}^{m}\right) \leqq \lim _{\alpha} \inf \omega_{\Lambda_{\alpha}}\left(N_{\Lambda}^{m}\right) \leqq c_{m}(\Lambda) .
$$

1 In applications to quantum gases the $N_{A}$ are local number operators and it suffices to have bounds for certain small values of $m$, i.e. $m \leqq 4$

(In N3, $m=1$ for Fermions, $m=3 / 2$ for Bosons, in N2 we need $m=2+\varepsilon$ for Fermions, $m=3+\varepsilon$ for Bosons) 
Further $\pi_{\omega}\left(N_{\Lambda}^{m}\right)$ is canonically defined, $\Omega_{\omega} \in D\left(\pi_{\omega}\left(N_{\Lambda}^{m / 2}\right)\right)$ and $\omega\left(N_{\Lambda}^{m}\right)=\left\|\pi_{\omega}\left(N_{\Lambda}^{m / 2}\right) \Omega_{\omega}\right\|^{2}$.

The principal result in the present context is the following

Theorem 2. Assume that conditions $\mathrm{H1}-\mathrm{H} 4, \mathrm{~N} 1-\mathrm{N} 3$, are fulfilled and, moreover, assume that the symmetric operator $H_{\omega}$, defined in Proposition 1, is essentially self-adjoint on $\pi_{\omega}(\mathfrak{B}) \Omega_{\omega}$.

It follows that the self-adjoint closure $\bar{H}_{\omega}$ of $H_{\omega}$ satisfies

1. $G(A, B ; t)=\left(\pi_{\omega}\left(A^{*}\right) \Omega_{\omega}, e^{i \bar{H}_{\omega} t} \pi_{\omega}(B) \Omega_{\omega}\right)$

for all $A, B \in \mathfrak{U}$

2. $e^{i \bar{H}_{\omega} t} \pi_{\omega}^{\prime \prime} e^{-i \bar{H}_{\omega} t}=\pi_{\omega}^{\prime \prime}, \quad t \in \mathbb{R}$,

and this group of automorphisms, is the $(\beta-)$ modular group associated with $\omega$ by Theorem 1.

The proof is divided into the following three lemmas.

Lemma 3. Adopt the assumptions $H 1-H 3$ and N1. Let $\Lambda_{n}$ denote the Greens functions $G$ on $\mathfrak{B} \times \mathfrak{B}$, i.e. $\Lambda_{n}$ is such that

$$
G(A, B ; t)=\lim _{n \rightarrow \infty} G_{\Lambda_{n}}(A, B ; t)
$$

for all $A, B \in \mathfrak{B}$.

Then $G$ satisfy the bounds

$$
|G(A, B ; t)|^{2} \leqq \omega\left(A A^{*}\right) \omega\left(B^{*} B\right)
$$

and hence can be extended by continuity to $\mathfrak{L} \times \mathfrak{L}$ where $\mathfrak{L}=\cup_{\Lambda} \mathfrak{L}\left(\mathfrak{H}_{\Lambda}\right)$. If $\Lambda_{\beta}$ is a subset of $\Lambda_{n}$ such that $\omega_{\Lambda_{\beta}}$ converges pointwise on $\mathfrak{Q}$ then

$$
G(A, B ; t)=\lim G_{\Lambda_{\beta}}(A, B ; t)
$$

for all $A, B \in \mathfrak{Q}$.

Proof. The bounds follow from Property 1 of the $G_{A}$ discussed at the beginning of the section.

Let $A \in \mathfrak{B}, B \in \mathfrak{L}$ and note that

$$
\begin{aligned}
& G_{\Lambda}(A, B ; t)-G(A, B ; t) \\
& =G_{\Lambda}\left(A, B-B^{\prime} ; t\right) \\
& \quad+G_{\Lambda}\left(A, B^{\prime} ; t\right)-G\left(A, B^{\prime} ; t\right)+G\left(A, B^{\prime}-B ; t\right)
\end{aligned}
$$

for any $B^{\prime} \in \mathfrak{B}$. Thus

$$
\begin{aligned}
& \left|G_{\Lambda}(A, B ; t)-G(A, B ; t)\right| \\
& \leqq \\
& \quad\left|G_{\Lambda}\left(A, B^{\prime} ; t\right)-G\left(A, B^{\prime} ; t\right)\right| \\
& \quad+\|A\| \omega_{\Lambda}\left(\left(B-B^{\prime}\right)^{*}\left(B-B^{\prime}\right)\right)^{1 / 2} \\
& \quad+\|A\| \omega\left(\left(B-B^{\prime}\right)^{*}\left(B-B^{\prime}\right)\right)^{1 / 2} .
\end{aligned}
$$


Thus

$$
\lim _{\beta} \sup \left|G_{A_{\beta}}(A, B ; t)-G(A, B ; t)\right| \leqq 2\|A\| \omega\left(\left(B-B^{\prime}\right)^{*}\left(B-B^{\prime}\right)\right)^{1 / 2} .
$$

Since $\pi_{\omega}(\mathfrak{B})$ is strongly dense in $\pi_{\omega}(\mathfrak{L})^{\prime \prime}$ the right hand side can be chosen arbitrarily small. Thus the desired result is obtained on $\mathfrak{B} \times \mathfrak{L}$. Repetition of the argument gives the same conclusion on $\mathfrak{L} \times \mathfrak{L}$.

Lemma 4. Adopt the assumptions $H 1-H 3$ and $N 1$. Let $N$ be a positive self-adjoint operator affiliated with $\mathfrak{Q}\left(\mathfrak{S}_{\Lambda_{N}}\right)$ and assume $\omega_{\Lambda}\left(N^{2}\right)<K$ uniformly for $\Lambda \supset \Lambda_{N}$. The extended Greens functions

$$
\begin{aligned}
G_{\Lambda}(A, B N ; t) & =\operatorname{Tr}_{\Lambda}\left(e^{-(\beta+i t) H_{\Lambda}} A e^{i t H_{\Lambda}} B N\right) / \operatorname{Tr}_{\Lambda}\left(e^{-\beta H_{\Lambda}}\right) \\
G(A ; B N ; t) & =\left(\pi_{\omega}\left(A^{*}\right) \Omega_{\omega}, X_{t} \pi_{\omega}(B N) \Omega_{\omega}\right)
\end{aligned}
$$

are well defined for all $A, B \in \mathfrak{Q}$.

If $\Lambda_{\beta}$ is the subnet of Lemma 3 then

$$
G(A, B N ; t)=\lim _{\beta} G_{\Lambda_{\beta}}(A, B N ; t)
$$

for all $A, B \in \mathfrak{Q}$ and $t \in \mathbb{R}$.

Proof. Since $\omega_{\Lambda}\left(N^{3}\right) \leqq K$ we have that

$$
\omega\left(N^{3}\right) \leqq \liminf _{\beta} \omega_{\Lambda_{\beta}}\left(N^{2}\right) \leqq K
$$

thus

$$
\begin{gathered}
\Omega_{\Lambda} \in D\left(\pi_{\omega_{\Lambda}}\left(N^{\frac{3}{2}}\right)\right) \subset D\left(\pi_{\omega_{\Lambda}}(N)\right) \\
\Omega \in D\left(\pi_{\omega}\left(N^{\frac{3}{2}}\right)\right) \subset D\left(\pi_{\omega}(N)\right)
\end{gathered}
$$

where $\Omega_{A}$ is the vector associated with $\omega_{A}$. Thus the extended Greens functions are well defined.

Let

$$
N=\int_{0}^{\infty} d E(\lambda) X
$$

be the spectral decomposition of $N$ and define

$$
N_{j}=\int_{0}^{j} d E(\lambda) \lambda \in \mathfrak{L}\left(\mathfrak{S}_{\Lambda_{N}}\right) .
$$

One has

$$
\begin{aligned}
G_{\Lambda} & (A, B N ; t)-G(A, B N ; t) \\
= & G_{\Lambda}\left(A, B\left(N-N_{j}\right) ; t\right) \\
& +G_{\Lambda}\left(A, B N_{j} ; t\right)-G\left(A, B N_{j} ; t\right) \\
& \quad-G\left(A, B\left(N-N_{j}\right) ; t\right) .
\end{aligned}
$$

The first and last terms can be bounded as follows

$$
\begin{aligned}
\left|G_{\Lambda}\left(A, B\left(N-N_{j}\right) ; t\right)\right|^{2} & \leqq \omega_{\Lambda}\left(A A^{*}\right) \omega_{\Lambda}\left(\left(N-N_{j}\right) B^{*} B\left(N-N_{j}\right)\right) \\
& \leqq\|A\|^{2}\|B\|^{2} \omega_{\Lambda}\left(N^{2}-N_{j}^{2}\right) .
\end{aligned}
$$


But one has

$$
\begin{aligned}
N^{2}-N_{j}^{2} & =\int_{j}^{\infty} d E(\lambda) \lambda^{2} \\
& \leqq 1 / j \int_{j}^{\infty} d E(\lambda) \lambda^{3} \\
& \leqq N^{3} / j .
\end{aligned}
$$

Combining these estimates gives

$$
\mid G_{A}\left(A, B\left(N-N_{j}\right) ; t \mid \leqq\|A\|\|B\| K^{1 / 2} / j^{1 / 2}\right.
$$

and, analogously

$$
\left|G\left(A, B\left(N-N_{j}\right) ; t\right)\right| \leqq\|A\|\|B\| K^{1 / 2} / j^{1 / 2} .
$$

Thus

$$
\begin{aligned}
& \left|G_{\Lambda_{\beta}}(A, B N ; t)-G(A, B N ; t)\right| \\
& \leqq \\
& \quad 2\|A\|\|B\| K^{1 / 2} / j^{1 / 2} \\
& \quad+\left|G_{\Lambda_{\beta}}\left(A, B N_{j} ; t\right)-G\left(A, B N_{j} ; t\right)\right| .
\end{aligned}
$$

Hence by Lemma 3

$$
\lim _{\beta} \sup G_{A_{\beta}}(A, B N ; t)-G\left(A, B N_{j} t\right) \mid \leqq 2\|A\|\|B\| K^{1 / 2} / j^{1 / 2} .
$$

But $j$ is arbitrary and the desired conclusion follows immediately.

Lemma 5. Adopt the assumptions $H 1-H 4, N 1-N 3$. For $B \in \mathfrak{B}$ choose $N_{A}^{m}$ as in N3. It follows that

$$
i H_{\omega} \pi_{\omega}(B) \Omega_{\omega}=\pi_{\omega}\left(\delta(B)\left(N_{\Lambda}^{m}+1\right)^{-1}\right) \pi_{\omega}\left(N_{\Lambda}^{m}+1\right) \Omega_{\omega}
$$

and for $A \in \mathfrak{B}$.

$$
\frac{d}{d t} G(A, B ; t)=G\left(A,\left(\delta(B)\left(N_{A}^{m}+1\right)^{-1}\right)\left(N_{A}^{m}+1\right) ; t\right) .
$$

Proof. By Lemma 1 one has

$$
\frac{d}{d t} G(A, B ; t)=\lim _{n \rightarrow \infty} \frac{d}{d t} G_{\Lambda_{n}}(A, B ; t)
$$

and using $\mathrm{H} 4$

$$
\begin{aligned}
\frac{d}{d t} G_{\Lambda_{n}}(A, B ; t) & =i G_{\Lambda_{n}}\left(A,\left[H_{\Lambda_{n}}, B\right] ; t\right) \\
& =G_{\Lambda_{n}}(A, \delta(B) ; t) \\
& =G_{\Lambda_{n}}\left(A,\left(\delta(B)\left(N^{m}+1\right)^{-1}\right)\left(N^{m}+1\right) ; t\right) .
\end{aligned}
$$

Hence by Lemma 4 applied to $N=N_{\Lambda}^{m}+1$ one has

$$
\begin{aligned}
\frac{d}{d t} G(A, B ; t) & =\lim _{\beta} G_{\Lambda_{\beta}}\left(A,\left(\delta(B)\left(N_{\Lambda}^{m}+1\right)^{-1}\right)\left(N_{A}^{m}+1\right) ; t\right) \\
& =G\left(A,\left(\delta(B)\left(N_{\Lambda}^{m}+1\right)^{-1}\right)\left(N_{\Lambda}^{m}+1\right) ; t\right) .
\end{aligned}
$$


This proves the last part of Lemma 4. The first part follows from the definition of $H_{\omega}$.

Proof of Theorem 2. If $B \in \mathfrak{B}$ then Lemma 5 implies

$$
\frac{d}{d t} X_{t} \pi_{\omega}(B) \Omega_{\omega}=i X_{t} H_{\omega} \pi_{\omega}(B) \Omega_{\omega}
$$

This implies that

$$
\begin{aligned}
\left(\pi_{\omega}(A) \Omega_{\omega}, \frac{d X_{t}}{d t} \pi_{\omega}(B) \Omega_{\omega}\right) & =\frac{d}{d t}\left(X_{-t} \pi_{\omega}(A) \Omega_{\omega}, \pi_{\omega}(B) \Omega_{\omega}\right) \\
& =i\left(X_{-t} H_{\omega} \pi_{\omega}(A) \Omega_{\omega}, \pi_{\omega}(B) \Omega_{\omega}\right) \\
& =i\left(H_{\omega} \pi_{\omega}(A) \Omega_{\omega}, X_{t} \pi_{\omega}(B) \Omega_{\omega}\right)
\end{aligned}
$$

for all $A, B \in \mathfrak{B}$. But the left hand side is continuous in $\pi_{\omega}(A) \Omega_{\omega}$ and this set of elements forms a core for $H_{\omega}$. Thus

$$
X_{t} \pi_{\omega}(B) \Omega_{\omega} \in D\left(H_{\omega}\right)
$$

and

$$
\begin{aligned}
\frac{d}{d t} X_{t} \pi_{\omega}(B) \Omega_{\omega} & =i H_{\omega} X_{t} \pi_{\omega}(B) \Omega_{\omega} \\
& =i X_{t} H_{\omega} \pi_{\omega}(B) \Omega_{\omega}
\end{aligned}
$$

for all $B \in \mathfrak{B}$. These equations have the solution $X_{t}=\exp \left\{i H_{\omega} t\right\}$ but this solution is unique because if $X_{t}$ is another solution one has

$$
\begin{aligned}
\frac{d}{d t} e^{-i H_{\omega} t} X_{t} \pi_{\omega}(B) \Omega_{\omega} & =i e^{i H_{\omega} t}\left\{-H_{\omega} X_{t}+X_{t} H_{\omega}\right\} \pi_{\omega}(B) \Omega_{\omega} \\
& =0, \quad B \in \mathfrak{B} .
\end{aligned}
$$

Thus $X_{t}=\exp \left\{i H_{\omega} t\right\}$. Therefore the Greens functions $G$ determine a unitary group and this group is the modular group associated with $\omega$ as a result of the discussion preceding the theorem.

Remark 2. In systems where $\delta(B)$ is defined as a bounded operator or if $\delta(B)=$ $\lim _{\alpha} i\left[H_{A_{\alpha}}, B\right]$ is an element of $\mathfrak{U}$ then Theorem 2 is true without any of the assumptions $\mathrm{N} 1-\mathrm{N} 3$. On the other hand one can also discuss situations in which $\left[H_{\Lambda}, B\right]$ does not become independent of $\Lambda$. If, for example, the $\Lambda$ are subsets of $\mathbb{R}^{v},\left\{\Gamma_{i}\right\}$ is a partitioning of $\mathbb{R}^{v}$ into unit cells, there exist $c_{i}(B)$ such that

$$
\omega\left(\left(\sum_{i} c_{i}(B) N_{\Gamma i}^{m}\right)^{3}\right)<+\infty
$$

and

$$
\left(\left[H_{\Lambda_{1}}, B\right]-\left[H_{\Lambda_{2}}, B\right]\right)\left(\sum_{i} c_{i}(B) N_{\Gamma_{\imath}}^{m}+1\right)^{-1}
$$

is a Cauchy set of bounded operators then the above proof can be extended. 
Remark 3. Theorem 2 has a partial converse. If the family of operators $X$ determined by the Greens functions $G$ is a one-parameter unitary group on $\mathfrak{H}_{\omega}$ then $H_{\omega}$ has equal deficiency indices. This follows by noting that the self-adjoint generator of $X$ extends $H_{\omega}$.

Remark 4. If, under the assumptions of Theorem $1, \Lambda_{n}$ is a subsequence such that the limits

$$
G(A, B ; t)=\lim _{n \rightarrow \infty} G_{\Lambda_{n}}(A, B ; t)
$$

exist for all $A, B \in \mathfrak{B}$ and $t \in \mathbb{R}$, and if, further, the one-parameter family of operators $X_{t}$ on $\mathfrak{H}_{\omega}$ determined by $G$ is unitary then the limits

$$
\begin{aligned}
& G\left(A, B_{1}, B_{2} \ldots B_{m} ; t_{1}, \ldots, t_{m}\right) \\
& =\lim _{n \rightarrow \infty} \operatorname{Tr}_{\Lambda_{n}}\left(e^{\beta H_{\Lambda_{n}}} A e^{i t_{1} H_{\Lambda_{n}}} B_{1} e^{-i\left(t_{1}-t_{2}\right) H_{\Lambda_{n}}} \ldots B_{m} e^{-i t_{m} H_{\Lambda_{n}}}\right) / \operatorname{Tr}_{\Lambda_{n}}\left(e^{-\beta H_{\Lambda_{n}}}\right)
\end{aligned}
$$

exist for all $A, B_{1}, \ldots, B_{m} \in \mathfrak{B}, t_{1}, \ldots, t_{m} \in \mathbb{R}$, and $m \in \mathbb{Z}_{+}$. Moreover

$$
\begin{aligned}
G\left(A, B_{1}, B_{2}, \ldots, B_{m} ; t_{1}, \ldots, t_{m}\right)= & \left(\Omega_{\omega}, \pi_{\omega}(A) X_{t_{1}} \pi_{\omega}\left(B_{1}\right) X_{t_{2}-t_{1}} \pi_{\omega}\left(B_{2}\right) \ldots X_{t_{n}-t_{n-1}} .\right. \\
& . . \Omega_{\omega}\left(B_{m}\right) \Omega_{\omega} \\
= & \omega\left(A \sigma_{t_{1}}\left(B_{1}\right) \ldots \sigma_{t_{m}}\left(B_{m}\right)\right)
\end{aligned}
$$

where $\sigma$ is the modular group of $\omega$. These statements follow from the foregoing discussion and Theorem 5 of [5]. For example pick any subsequence of the $A_{n}$ such that the limits $G\left(A, B_{1}, \ldots, B_{m} ; t_{1}, \ldots, t_{m}\right)$ exist for all $A_{1}, B_{1}, \ldots, B_{m} \in \mathfrak{B}$ etc. Such a sequence exists by the reasoning of [5]. By Theorem 5 of [5] there exists a Hilbert space $\mathfrak{K}_{\omega} \supset \mathfrak{H}_{\omega}$, a representation $\hat{\pi}_{\omega}$ of $\mathfrak{A}$, and a unitary group $U$ acting on $\mathfrak{R}_{\omega}$, such that

$$
G\left(A, B_{1}, \ldots, B_{m} ; t_{1}, \ldots, t_{m}\right)=\left(\Omega_{\omega}, \hat{\pi}_{\omega}(A) U_{t_{1}} \hat{\pi}_{\omega}\left(B_{1}\right) \ldots U_{t_{m}-t_{m-1}} \hat{\pi}_{\omega}\left(B_{m}\right) \Omega_{\omega}\right) .
$$

Now if $P$ is the orthogonal projector on $\mathfrak{K}_{\omega}$ with range $\mathfrak{H}_{\omega}$ we deduce that $X_{t}=$ $P U_{t} P$. Hence if $X_{t}$ is unitary then $U_{t}$ commutes with $P$ because

$$
\left(P U_{t} P\right)^{*}\left(P U_{t} P\right)-P=\left(P U_{t}(1-P)\right) *\left(P U_{t}(1-P)\right)
$$

and hence $P U_{t} P$ is unitary on $\mathfrak{G}_{\omega}$ if, and only if, $P U_{t}(1-P)=0$. But $\hat{\pi}_{\omega}$ is an extension of $\pi_{\omega}$ such that $\hat{\pi}_{\omega} \Omega_{\omega}=\pi_{\omega} \Omega_{\omega}$ and $\Omega_{\omega}$ is cyclic for $\left\{\pi_{\omega}, U_{\omega}\right\}$ in $\Omega_{\omega}$. Hence $\mathfrak{R}_{\omega}=\mathfrak{H}_{\omega}$, $P=1$, and $X_{t}=U_{t}$. These equalities identify $G$ and simultaneously prove that each convergent subsequence of the $G_{\Lambda_{n}}$ gives the same limit, i.e. the limit of the $G_{\Lambda_{n}}$ exists.

\section{Applications}

In this section we discuss the applications of the abstract theorems of Section 2 to long range spin systems $[5,8]$ and low density Fermi and Bose gases $[6,9]$.

\section{a) Quantum Spin Systems}

We adopt the notation and assumptions of [5] for spin systems. The quasi-local algebra $\mathfrak{U}$ associated with the system is norm separable. 
If $\Phi=\{\Phi(X)\}_{X \subset \mathbb{Z}^{v}}$ is an interaction then

$H_{\Lambda}=\sum_{X \subset \Lambda} \Phi(X) \in \mathfrak{U}_{\Lambda}$

is bounded and conditions $\mathrm{H} 1-\mathrm{H} 2$ are trivially satisfied. If the interaction $\Phi$ satisfies the condition of [5]

$$
\sup _{x \in \mathbb{Z}^{\nu}} \sum_{X \ni x}\|\Phi(X)\|<+\infty
$$

then for $B \in \mathfrak{U}_{\Lambda}$

$$
\begin{aligned}
\delta(B) & =\lim _{\Lambda \rightarrow \infty} i\left[H_{\Lambda}, B\right] \\
& =\sum_{X \Lambda \Phi} i[\Phi(X), B]
\end{aligned}
$$

and $\mathrm{H} 3$ is trivially satisfied. Thus Theorem 1 is applicable.

Although $\left[H_{A}, B\right]$ does not become independent of $A$ the limit $\delta(B)$ is bounded and this replaces condition $\mathrm{H} 4$. (The conditions N1-N3 are unnecessary in this case.) Thus Theorem 2 applies.

Every equilibrium state of the long range spin systems considered in [5] is a modular state.

For a particular subclass of these systems Pulvirenti and Tirozzi [8] have established that the Hamiltonian $H_{\omega}$ of Proposition 1 is essentially self-adjoint and hence the Greens functions and the modular automorphisms are directly related. These authors prove the essential self-adjointness by showing that $H_{\omega}$ has a dense set of analytic vectors.

\section{b) Dilute Fermi Gas}

Low density Fermi gases with two body interactions have been studied in [6]. We adopt the notation of this reference. The $C^{*}$-algebra $\mathfrak{A}$, the CAR algebra, associated with these systems is norm-separable. It is established that if the interaction is mediated by a stable two-body potential $\phi \in L^{2}\left(\mathbb{R}^{v}\right) \cap L^{1}\left(\mathbb{R}^{v}\right)$ which is even and continuous for $x \neq 0$ then conditions $\mathrm{H} 1-\mathrm{H} 3$ are satisfied for a suitable choice of the $\mathfrak{D}_{\Lambda}$, whenever the activity is sufficiently small. For example one may choose $\mathfrak{D}_{\Lambda}$ to be the algebra generated by creation and annihilation operators $a^{*}(f), a(g)$, with $f$ and $g$ twice continuously differentiable functions in $L^{2}(\Lambda)$. Theorem 1 applies with $\mathfrak{B}_{\Lambda}=\mathfrak{D}_{\Lambda}$. The results of [6] are based on the work of Ginibre [14] who establishes that at small activity and with fixed boundary conditions there is a unique limit of the Gibbs states $\omega_{\Lambda}$. Therefore the small activity Gibbs state is a modular state.

If next we choose the interaction $\phi$ to have compact support then condition $\mathrm{H} 4$ is also satisfied but $\delta(B)$ is unbounded for $B \in \mathfrak{B}_{A}$. In the appendix we give estimates of the $\delta(B)$ in terms of the particle number operators $N_{A}$ associated with the regions $\Lambda \in \mathbb{R}^{v}$. In particular we show that for suitable $\Lambda_{B}$

$$
\left\|\delta(B)\left(N_{A_{B}}+1\right)^{-1}\right\|<+\infty .
$$

Thus condition N3 is satisfied. 
It remains to check conditions $\mathrm{N} 1-\mathrm{N} 2$. In the small fugacity region studied by Ginibre [14] one has, however, that the local Gibbs states $\omega_{A}$ converge in the weak* topology of $\mathfrak{A}$ to a locally normal state $\omega$. Moreover the density matrices $\varrho_{\Lambda, \Lambda_{0}}$ obtained by restricting $\omega_{\Lambda}$ to $\mathfrak{I}_{\Lambda_{0}}$, i.e. the density matrices such that

$$
\omega_{\Lambda}(A)=\operatorname{Tr}_{\mathfrak{H}_{\Lambda_{0}}}\left(\varrho_{\Lambda, \Lambda_{0}} A\right), \quad A \in \mathfrak{A}_{\Lambda_{0}},
$$

converge strongly on $\mathfrak{H}_{\Lambda_{0}}$ to the density matrices $\varrho_{\Lambda_{0}}$ associated with $\Omega$ (see, for example, [14] Proposition 3.3). But this implies that $\varrho_{\Lambda, \Lambda_{0}}$ converges to $\varrho_{\Lambda_{0}}$ in the trace-norm topology (see, for example, [19] and [15] Corollary 1) and hence $\omega_{A}$ converges to $\omega$ uniformly on each $\mathfrak{Q}\left(\mathfrak{H}_{A_{0}}\right)$. Thus condition N1 is valid.

Finally condition $\mathrm{N} 2$ follows in the small fugacity region with $N_{A}$ chosen as the number operator (see appendix) because the correlation functions $\varrho_{A}(X, Y)$ associated with the $\omega_{A}$ are bounded ([14] Chapter 3) uniformly in $\Lambda$.

But one has

$$
\begin{aligned}
& \omega_{\Lambda}\left(N_{\Lambda_{0}}\left(N_{\Lambda_{0}}+1\right) \ldots\left(N_{\Lambda_{0}}+m-1\right)\right) \\
& =\int_{\Lambda_{0}^{m}} d x_{1} \ldots d x_{m} \varrho_{\Lambda}\left(x_{1} \ldots x_{m} ; x_{m} \ldots x_{1}\right) .
\end{aligned}
$$

Hence we may conclude that Theorem 2 is applicable to the interacting Fermi gas at low fugacity if the two-body potential $\phi$ is finite range, $\phi \in L^{2}\left(\mathbb{R}^{v}\right) \cap L^{1}\left(\mathbb{R}^{v}\right)$, $\phi$ is stable, even and continuous for $x \neq 0$.

\section{c) Dilute Bose Gas}

Results similar to those of [6] for the fermi gas have been obtained in [9] for the Bose gas. The $C^{*}$-algebra appropriate to the description of the Bose gas is the algebra associated to the canonical commutation relations, the CCR algebra $\mathfrak{A}$. This algebra is generated by unitary elements $U(f), V(g)$, the Weyl operators which are defined for each pair $f, g \in L^{2}\left(\mathbb{R}^{v}\right)$. The algebra is not norm separable. In the small fugacity region there again exists a unique Gibbs limit state $\omega$ for each fixed boundary condition and this state is locally normal. These statements again follow from [14]. Thus there exists a separable subalgebra $\mathfrak{B} \subset \mathfrak{A}$ such that the restriction of $\omega$ to $\mathfrak{B}$ fully determines $\omega$. One can consider the *-algebra $\mathfrak{B}$ where the corresponding local algebra $\mathfrak{B}_{A}$ are generated by Weyl operators $U(f), V(g)$ with $f, g$ chosen in some fixed orthonormal basis of $L^{2}(\Lambda)$. If, further, the basis of $L^{2}(\Lambda)$ are chosen to be formed of twice differentiable functions then the estimates of [9] establish conditions $\mathrm{H} 1-\mathrm{H} 3$ in the small fugacity region for an interaction of the type previously considered for fermions. Thus Theorem 1 is applicable. Under similar conditions Theorem 2 can be applied if the interaction is taken to have finite range. Verification of conditions H4 and N1-N3 is similar to the Fermi case and relies on the results of [14] and the estimates of the appendix.

\section{d) Bose Gas with Repulsive Interactions}

As a final application we consider the Bose gas with a positive two-body interaction. The density estimates which are necessary for the application of Theorem 2 have recently been obtained by Suhov [17]. 
Proposition 2 (Suhov). Let $\mathfrak{H}_{F}(\Lambda), \Lambda \subset \mathbb{R}^{v}$, be the Fock space appropriate to the description of Bose particles confined to $\Lambda$ and $T_{\Lambda}^{D}$, $T_{\Lambda}^{N}$ the kinetic energy operators with Dirichlet and Neumann boundary conditions respectively. If $x \in \mathbb{R}^{v} \rightarrow \phi(x)$ is a positive twice continuously differentiable function such that $\phi^{\prime}$ is integrable and $\phi^{\prime \prime}$ is bounded, and $U_{\phi}(\Lambda)$ the associated interaction operator on $\mathfrak{H}_{F}(\Lambda)$ we define the total Hamiltonian $H_{\Lambda}$ as the Friederichs extension of $T_{A}^{D}+U_{\phi}(\Lambda)$. Further we denote the number operator on $\mathfrak{H}_{F}(\Lambda)$ by $N_{\Lambda}$ and for $\beta>0, \mu<0$, we define the Gibbs state $\omega_{\beta, \mu, \Lambda}$ by

$$
\omega_{\beta, \mu, \Lambda}(A)=\operatorname{Tr}_{\mathfrak{H}_{F(A)}}\left(e^{-\beta\left(H_{\Lambda}-\mu N_{\Lambda}\right)} A\right) / \operatorname{Tr}_{\mathfrak{H}_{F(A)}}\left(e^{-\beta\left(H_{\Lambda}-\mu N_{A}\right)}\right)
$$

for all $A \in \mathfrak{Q}\left(\mathfrak{S}_{F}(\Lambda)\right)$.

It follows that for $\Lambda_{0} \subset \Lambda$

$$
\begin{aligned}
& \omega_{\beta, \mu, \Lambda}\left(N_{\Lambda_{0}}^{m}\right) \leqq c_{m}\left(\Lambda_{0}\right)<+\infty, m=1,2, \ldots \\
& \omega_{\beta, \mu, \Lambda}\left(T_{\Lambda_{0}}^{N}\right) \leqq c\left(\Lambda_{0}\right)<+\infty
\end{aligned}
$$

where $c_{m}$ c are independent of $\Lambda$.

This proposition allows us to make our principal application

Corollary 1. Adopt the framework of Proposition 2 but further assume that the interaction $\phi$ has compact support and $\phi \in L^{2}\left(\mathbb{R}^{v}\right)$. Let $\omega_{\beta, \mu, \Lambda_{\alpha}}$ be a weak ${ }^{*}$ convergent subnet and $\omega_{\beta, \mu}$ the corresponding weak* limit point.

It follows that

1. $\omega_{\beta, \mu}$ is locally normal;

2. $\omega_{\beta, \mu, \Lambda_{\alpha}}$ converges in norm to $\omega_{\beta, \mu}$ on $\mathfrak{L}\left(\mathfrak{H}_{F}(\Lambda)\right)$ for each $\Lambda$;

3. $\omega_{\beta, \mu}$ is a modular state on the von Neumann algebra generated by $\cup_{\Lambda} \mathfrak{L}\left(\mathfrak{S}_{F}(\Lambda)\right)$;

4. if the thermodynamic $H_{\omega}$, associated with $\omega_{\beta, \mu}$ by Proposition 1 is essentially self-adjoint then its closure $\bar{H}_{\omega}$ is the generator of the modular group of $\omega_{\beta, \mu}$.

Proof. The first statement is a consequence of the first density estimate of Proposition 2 and Corollary 1 of [17]. The second statement follows from the energy estimate of Proposition 2 and Theorem 3 of [15]. Because $T_{\Lambda_{0}}^{N}+N_{\Lambda_{0}}$ has a compact resolvent on $\mathfrak{H}_{F}\left(\Lambda_{0}\right)$. The third statement follows by application of Theorem 1 . Note that conditions $\mathrm{H} 1-\mathrm{H} 3$ are valid by the estimates of the appendix combined with Proposition 2 . In this context we again take $\mathfrak{B}_{\Lambda}$ to be the *-algebra generated by Weyl operators $U(f), V(g)$ with $f, g$ chosen to be twice differentiable and in some fixed orthonormal basis of $L^{2}(\Lambda)$. Finally condition $\mathrm{H} 4$ is valid because $\phi$ has compact support, $\mathrm{N} 1$ follows from the first statements of the Corollary, N2 is a consequence of Proposition 2, and N3 is established in the appendix. Thus the fourth statement follows from Theorem 2. Finally we note that the above result, Corollary 1 , also holds for one-dimensional systems with a hard core and finite range $C^{2}$-interaction [20].

\section{Appendix}

In this appendix we derive a number of estimates for commutators of Hamiltonian operators and field operators acting on Fock space. 
We let $\mathfrak{H}_{F}$ denote the usual Fock space built over $L^{2}\left(\mathbb{R}^{v}\right)$, either symmetrically or anti-symmetrically, and $a(f), a^{*}(f)$ the usual annihilation and creation operators, defined for each real $f \in L^{2}\left(\mathbb{R}^{v}\right)$. If $\Lambda$ is a bounded open subset of $\mathbb{R}^{v}$ we define the local number operator $N_{A}$ by

$$
\left(N_{\Lambda} \psi\right)^{(n)}\left(x_{1} \ldots x_{n}\right)=\sum_{i=1}^{n} \chi_{\Lambda}\left(x_{i}\right) \psi^{(n)}\left(x_{1}, \ldots, x_{n}\right)
$$

where $\chi_{\Lambda}$ is the characteristic function of $\Lambda$ and $\psi=\left\{\psi^{(n)}\right\} \in \mathfrak{H}_{F}$ is such that $\psi^{(n)}=0$ for $n$ sufficiently large. $N_{A}$ is symmetric and its closure, which we also denote by $N_{\Lambda}$, is self-adjoint.

If $\phi$ is a real symmetric function then we define the associated interaction operator $U_{\phi}$ on $\mathfrak{H}_{F}$ by

$$
\left(U_{\phi} \psi\right)^{(n)}\left(x_{1} \ldots x_{n}\right)=\sum_{i \neq j} \phi\left(x_{i}-x_{j}\right) \psi^{(n)}\left(x_{1} \ldots x_{n}\right) .
$$

Actually we need further conditions on $\phi$ to ensure that the foregoing formal definition specifies an operator on $\mathfrak{H}_{F}$ and we should also be more precise concerning the domain of this operator. These points will be clarified in each of the results given below, e.g. $\phi \in L^{2}\left(\mathbb{R}^{v}\right)$ will specify an operator $U_{\phi}$ with domain containing $D\left(N_{\Lambda}^{2}\right)$ for all $\Lambda$.

Lemma A1. Take $f \in L^{2}(\Lambda)$ and $\phi \in L^{2}\left(\Lambda^{\prime}\right)$ then

$$
\begin{aligned}
\left\|\left[U_{\phi}, a(f)\right] \psi\right\| & \leqq\|\phi\|_{2}\left\|a(f)\left(N_{\Lambda-\Lambda^{\prime}}-1\right) \psi\right\| \\
& \leqq\|\phi\|_{2}\|f\| X\left(N_{\Lambda-\Lambda^{\prime}}-1\right) \psi \|
\end{aligned}
$$

where

$$
\begin{aligned}
X & =1 \text { for fermions (anti-symmetry) } \\
& =N_{\Lambda}^{1 / 2} \quad \text { for bosons (symmetry) }
\end{aligned}
$$

and $\Lambda-\Lambda^{\prime}=\left\{x \in \mathbb{R}^{v} ; x=y-z, y \in \Lambda, z \in \Lambda^{\prime}\right\}$.

Proof. Using the action of annihilation operators, and the definition of $U_{\phi}$, one immediately calculates that

$$
\begin{aligned}
\left\|\left[U_{\phi}, a(f)\right] \psi\right\|^{2}= & \sum_{n \geqq 0}(n+1) \int d x_{1} \ldots d x_{n} \int d x d y f(x) f(y) \sum_{i=1}^{n} \phi\left(x-x_{i}\right) \sum_{j=1}^{n} \phi\left(y-x_{j}\right) \\
& \psi^{(n+1)}\left(x, x, \ldots, x_{n}\right)^{-} \psi^{(n+1)}\left(y, x, \ldots, x_{n}\right) .
\end{aligned}
$$

We now sum the right hand side over a complete orthonormal basis of $L^{2}\left(\Lambda^{\prime}\right)$ which is chosen to contain $\phi /\|\phi\|_{2}$. This gives the inequality

$$
\begin{aligned}
\left\|\left[U_{\phi}, a(f)\right] \psi\right\|^{2} \leqq & \|\phi\|_{2}^{2} \sum_{n \geqq 0}(n+1) \sum_{i, j} \int d x \int_{x-x_{i} \in \Lambda^{\prime}, x-x_{j} \in \Lambda^{\prime}} d x, \ldots, d x_{n} \\
& \cdot f(x) f\left(x-x_{i}+x_{j}\right) \\
& \psi^{(n+1)}\left(x_{1} x_{1} \ldots x_{n}\right)^{-} \psi^{(n+1)}\left(x-x_{i}+x_{j}, x_{1} \ldots x_{n}\right) .
\end{aligned}
$$


Applying the Schwartz inequality one then finds

$$
\begin{aligned}
\left\|\left[U_{\phi}, a(f)\right] \psi\right\|^{2} & \leqq\left.\|\phi\|_{2}^{2} \sum_{n \geqq 0} \sum_{i j} \int_{x_{i}, x_{j} \in \Lambda-\Lambda^{\prime}} d x_{1} \ldots d x_{n}(a(f) \psi)^{(n)}\left(x_{1} \ldots x_{n}\right)\right|^{2} \\
& =\|\phi\|_{2}^{2}\left\|N_{\Lambda+\Lambda^{\prime}} a(f) \psi\right\|^{2} \\
& =\|\phi\|_{2}^{2}\left\|a(f)\left(N_{\Lambda+\Lambda^{\prime}}-1\right)\right\|^{2} .
\end{aligned}
$$

Finally for fermions one has

$$
a^{*}(f) a(f) \leqq\|f\|_{2}^{2}
$$

and for bosons

$$
a^{*}(f) a(f) \leqq\|f\|_{2}^{2} N_{\Lambda} .
$$

Lemma A2. Take $f \in L^{2}(\Lambda)$ and $\phi \in L^{2}\left(\Lambda^{\prime}\right)$ with $0 \in \Lambda^{\prime}$ then

$$
\begin{aligned}
\left\|\left[U_{\phi}, a^{*}(f)\right] \psi\right\| & \leqq\|\phi\|_{2}\|f\|_{2}\left\|N_{\Lambda-\Lambda^{\prime}} \psi\right\| \text { for fermions } \\
& \leqq\|\phi\|_{2}\|f\|_{2}\left\|\left(N_{\Lambda-\Lambda^{\prime}}+1\right)^{1 / 2} N_{\Lambda-\Lambda^{\prime}} \psi\right\| \text { for bosons } .
\end{aligned}
$$

Proof. First note that

$$
\mathfrak{H}_{F}=\mathfrak{H}_{F}(\Lambda) \otimes \mathfrak{H}_{F}\left(\Lambda^{c}\right), \quad \Lambda \subset \mathbb{R}^{v},
$$

where $\Lambda^{c}$ denotes the complement of $\Lambda$ in $\mathbb{R}^{v}$ and $\otimes$ denotes the anti-symmetrized or symmetrized tensor product according to the choice of statistics. As all operators occuring in the statement of the Lemma act on $\mathfrak{H}_{F}\left(\Lambda-\Lambda^{\prime}\right)$ it suffices to prove the inequalities on this latter space.

In Lemma A1 we established that

$$
\left\|\left[U_{\phi}, a(f)\right]\left(N_{A-\Lambda^{\prime}}-1\right)^{-1}\right\| \leqq\|f\|_{2}\|\phi\|_{2}
$$

for fermions and, using $\Lambda \subset \Lambda-\Lambda^{\prime}$ and hence $N_{\Lambda} \leqq N_{\Lambda-\Lambda^{\prime}}$,

$$
\left\|\left[U_{\phi}, a(f)\right]\left(N_{\Lambda-\Lambda^{\prime}}-1\right)^{-1} N_{\Lambda-\Lambda^{\prime}}{ }^{1 / 2}\right\| \leqq\|f\|_{2}\|\phi\|_{2}
$$

for bosons. Taking adjoints and using the fact that $\left[U_{\phi}, a^{*}(f)\right]$ maps the $n$-particle subspace of $\mathfrak{H}_{F}\left(\Lambda-\Lambda^{\prime}\right)$ onto the $n+1$-particle subspace gives

$$
\left\|\left[U_{\phi}, a^{*}(f)\right] N_{\Lambda-\Lambda^{\prime}}-1\right\| \leqq\|f\|_{2}\|\phi\|_{2}
$$

for fermions and

$$
\left\|\left[U_{\phi}, a^{*}(f)\right] N_{\Lambda-N}\left(N_{\Lambda-\Lambda^{\prime}}+1\right)^{1 / 2}\right\| \leqq\|f\|_{2}\|\phi\|_{2}
$$

for bosons.

In the case of bosons it is useful to consider bounded functions of the unbounded operators $a(f), a^{*}(f)$. Let $\phi(f)$ and $\pi(f)$ denote the usual self-adjoint field operators associated with the $a(f)$ and $a^{*}(f)$, e.g.

$$
\phi(f)=2^{-1 / 2}\left(a(f)+a^{*}(f)\right)
$$

and let $W(f, g)$ be the unitary operators given by

$$
W(f, g)=\exp \{i \phi(f)+i \pi(g)\} .
$$


Lemma A3. Take $f, g \in L^{2}(\Lambda)$ and $\phi \in L^{2}\left(\Lambda^{\prime}\right)$ with $0 \in \Lambda^{\prime}$ then

$$
\begin{gathered}
\left\|\left[U_{\phi}, W(f, g)\right] \psi\right\| \leqq \\
\|\phi\|_{2}\left(\|f\|_{2}+\|g\|_{2}\right)\left(a_{0}+a_{1}\left(\|f\|_{2}+\|g\|_{2}\right)\right. \\
\end{gathered}
$$

where $a_{0}, a_{1}$ are positive constants independent of $f, g, \phi, \psi$.

Proof. One has

$$
\begin{aligned}
W(f, g)^{*}\left[U_{\phi}, W(f, g)\right] & =W^{*}(f, g) U_{\phi} W(f, g)-U_{\phi} \\
& =i \int_{0}^{1} d s W^{*}(s f, s g)\left[U_{\phi}, \phi(f)+\pi(g)\right] W(s f, s g) .
\end{aligned}
$$

Therefore

$$
\left\|\left[U_{\phi}, W(f, g)\right] \psi\right\| \leqq \int_{0}^{1} d s\left\|\left[U_{\phi}, \phi(f)+\pi(g)\right] W(s f, s g) \psi\right\| .
$$

But $\phi$ and $\pi$ are linear combinations of $a$ and $a^{*}$. Hence applying Lemma A2

$$
\left\|\left[U_{\phi}, W(f, g)\right] \psi\right\| \leqq\|\phi\|_{2}\left(\|f\|_{2}+\|g\|_{2}\right) 2 \int_{0}^{1} d s\left\|\left(N_{\Lambda-\Lambda^{\prime}}+1\right)^{\frac{3}{2}} W(s f, s g) \psi\right\| .
$$

Next we use

$$
W^{*}(s f, s g) N_{\Lambda-\Lambda^{\prime}} W(s f, s g)=\hat{N}_{\Lambda-\Lambda^{\prime}}
$$

where

$$
\hat{N}_{\Lambda-\Lambda^{\prime}}=N_{\Lambda-\Lambda^{\prime}}+s(\pi(f)-\phi(g))+\frac{s^{2}}{2}\left(\|f\|_{2}^{2}+\|g\|_{2}^{2}\right) .
$$

Thus

$$
\left\|\left[U_{\phi}, W(f, g)\right] \psi\right\| \leqq\|\phi\|_{2}\left(\|f\|_{2}+\|g\|_{2}\right) 2 \int_{0}^{1} d s\left\|\left(\hat{N}_{\Lambda-\Lambda^{\prime}}+1\right)^{\frac{3}{2}} \psi\right\| .
$$

Finally we bound $\left(\hat{N}_{\Lambda-\Lambda^{\prime}}+1\right)^{3}$ in terms of $\left(N_{\Lambda-\Lambda^{\prime}}+1\right)^{3}$ by using the commutation relations of $N_{\Lambda-\Lambda}$ with $\phi$ and $\pi$ and the bounds

$$
\begin{aligned}
\phi(f)^{2} & \leqq 2\left(N_{\Lambda}+1\right)\|f\|^{2} \\
\phi(f) & \leqq 2^{1 / 2}\left(N_{\Lambda}+1\right)^{1 / 2}\|f\|_{2} \leqq 2^{1 / 2}\left(N_{\Lambda}+1\right)\|f\|_{2} \\
N_{\Lambda} & \leqq N_{\Lambda-\Lambda^{\prime}}
\end{aligned}
$$

etc. The last inequality is a consequence of our assumption that $\Lambda^{\prime} \ni 0$.

Combination of these inequalities yields a result of the form stated.

These estimates can be extended to certain potential functions $\phi$ which do not have compact support.

Lemma A4. Let $\psi \in \mathfrak{H}_{F}$ be such that for every $\Lambda \subset \mathbb{R}^{v}$ one has $\psi \in D\left(N_{A}^{m}\right)$ and

$$
\left\|N_{\Lambda}^{m} \psi\right\|<|\Lambda|^{m} c_{\psi}
$$

where $m=1$ for fermions and $m=3 / 2$ for bosons, and $c_{\psi}$ is independent of $\Lambda$. Further let $f \in L^{2}\left(\Lambda_{f}\right)$ for some $\Lambda_{f}$ and take $\phi$ such that

$$
|\phi|_{\varepsilon}^{2}=\int d^{v} x\left(1+|x|^{2}\right)^{v+\varepsilon}|\phi(x)|^{2}<+\infty
$$

for some $\varepsilon>0$. 
It follows that

$$
\left\|\left[U_{\phi}, a^{\#}(f)\right] \psi\right\| \leqq d\left(c_{\psi}, \Lambda_{f}, \varepsilon\right)|\phi|_{\varepsilon}\|f\|_{2}
$$

where $a^{\#}$ denotes either a or $a^{*}$, and $d$ is a constant. Further, for bosons, with $f \in L^{2}\left(\Lambda_{f}\right), g \in L^{2}\left(\Lambda_{g}\right)$

$$
\begin{gathered}
\|\left[U_{\phi}, W(f, g) \mid \psi \| \leqq\right. \\
\left(c_{\psi}, \Lambda_{f}, \Lambda_{g}, \varepsilon\right)|\phi|\left(\|f\|_{2}+\|g\|_{2}\right) \\
\left.\left(\|f\|_{2}+\left\|g_{2}\right\|\right)\right)
\end{gathered}
$$

where $a_{0}, a_{1}, d$ are constants.

Proof. Partition $\mathbb{R}^{v}$ into a cubic lattice with unit cells $\Lambda_{i}$ and define $\phi_{i}=\chi_{\Lambda_{i}} \phi$. One has

$$
\begin{aligned}
\left\|\left[U_{\phi}, a^{\#}(f)\right] \psi\right\| & \leqq \sum_{i}\left\|\left[U_{\phi_{i}}, a^{\#}(f)\right] \psi\right\| \\
& \leqq \sum_{i}\|f\|_{2}\left\|\phi_{i}\right\|_{2}\left\|N_{\Lambda_{f}-\Lambda_{i}}^{m} \psi\right\| \\
& \leqq \sum_{i}\|f\|_{2}\left\|\phi_{i}\right\|_{2} c_{\psi}\left|\Lambda_{f}-\Lambda_{i}\right|^{m}
\end{aligned}
$$

where the first step follows from the triangle inequality, the second is an application of Lemma A1, or Lemma A2, and the third follows from the hypothesis on 4 . Next note that $\left|\Lambda_{f}-\Lambda_{i}\right|$ is uniformly bounded by a constant $d_{f}$ and

$$
|\phi|_{\varepsilon}^{2} \geqq a_{i}\left\|\phi_{i}\right\|^{2}
$$

where

$$
a_{i}=\min _{x \in \Lambda_{i}}\left(1+x^{2}\right)^{v+\varepsilon} .
$$

Thus

$$
\sum_{i}\left\|\phi_{i}\right\| \leqq|\phi|^{\varepsilon} \sum_{i} a_{i}^{-1 / 2} .
$$

Combining these estimates one has

$$
\left\|\left[U_{\phi}, a^{\#}(f)\right] \psi\right\| \leqq c_{\psi} d_{f}^{m}\left(\sum_{i} a_{i}^{-1 / 2}\right)\|f\|_{2}|\phi|_{\varepsilon} .
$$

The second statement of the lemma follows by an identical argument but with Lemma A3 replacing Lemmas A1 and A2.

Remark. A result similar to Lemma A4 is valid for states which are locally determined by density matrices $\varrho_{A}$ on $\mathfrak{H}_{F}(\Lambda)$ and such that

$$
\left|\operatorname{Tr}_{\mathfrak{H}_{F(\Lambda)}}\left(\varrho_{\Lambda} N_{\Lambda}^{m}\right)\right|<|\Lambda|^{m} c_{\varrho} .
$$

Next we turn to estimates involving the kinetic energy operator. Let $T$ denote the usual self-adjoint operator on $\mathfrak{H}_{F}$ whose action on the twice differentiable vectors $\psi=\left\{\psi^{(n)}\right\}$, with $\psi^{(n)}=0$ for large $n$, is given by

$$
(T \psi)^{(n)}\left(x_{1} \ldots x_{n}\right)=-\sum_{i=1}^{n} \nabla_{x_{\imath}}^{2} \psi^{(n)}\left(x_{1}, \ldots, x_{n}\right) .
$$


If $f$ is a twice-differentiable element of $L^{2}(\Lambda)$ one has

$$
\begin{aligned}
([T, a(f)] \psi)^{(n)}\left(x_{1} \ldots x_{n}\right) & =-\int d x f(x) \nabla_{x}^{2} \psi^{(n+1)}\left(x, x_{1} \ldots x_{n}\right) \\
& =-\left(a\left(\nabla^{2} f\right) \psi\right)^{(n)}\left(x_{1} \ldots x_{n}\right) .
\end{aligned}
$$

Thus

$$
\begin{aligned}
\|[T, a(f)] \psi\| & \leqq\left\|a\left(\nabla^{2} f\right) \psi\right\| \\
& \leqq\left\|\nabla^{2} f\right\|_{2}\|X \psi\|
\end{aligned}
$$

where

$$
\begin{aligned}
X & =1 \text { for fermions } \\
& =N_{\Lambda}^{1 / 2} \text { for bosons } .
\end{aligned}
$$

Similarly

$$
\left\|\left[T, a^{*}(f)\right] \psi\right\| \leqq\|Y \psi\|
$$

where

$$
Y=1 \text { for fermions }
$$$$
=\left(N_{\Lambda}+1\right)^{1 / 2} \text { for bosons . }
$$

Lemma A5. Let $f, g \in L^{2}(\Lambda)$ be twice differentiable then

$$
\begin{aligned}
\|[T, W(f, g)] \psi\| \leqq\left(\|\nabla f\|_{2}^{2}\right. & \left.+\|\nabla g\|_{2}^{2}\right)\|\psi\| \\
& +2\left(\left\|\nabla^{2} f\right\|_{2}+\left\|\nabla^{2} g\right\|_{2}\right)\left\|\left(N_{A}+1\right)^{1 / 2} \psi\right\| .
\end{aligned}
$$

Proof. One argues, as in the proof of Lemma A3, that

$$
\begin{aligned}
\|[T, W(f, g)] \psi\| & \leqq \int_{0}^{1} d s\|[\phi(f)+\pi(g), T] W(s f, s g) \psi\| \\
& =\int_{0}^{1} d s\left\|\left(\pi\left(\nabla^{2} f\right)-\phi\left(\nabla^{2} g\right)\right) W(s f, s g) \psi\right\| \\
& \left.=\int_{0}^{1} d s \|\left[\pi\left(\nabla^{2} f\right)-\phi\left(\nabla^{2} g\right)\right)+s\left(\|\nabla f\|^{2}+\|\nabla g\|^{2}\right)\right] \psi \| .
\end{aligned}
$$

One then uses $\pi(h)^{2} \leqq 2 N_{\Lambda}+1$ for $h \in L^{2}(\Lambda)$ etc.

Acknowledgements. This work was begun at the end of 1973 and initially discussions with A. Connes and M. Winnink were very useful. The work was continued in Spring 1974 whilst one author (DWR) was a guest of the Dept. of Physics at the University of Groningen and finished in Autumn 1975 whilst the other author (OB) was a guest of the Centre de Physique Theorique, CNRS, Marseille. We are both grateful to the members of the respective centres for their hospitality.

\section{References}

1. Robinson, D. W.: Commun. math. Phys. 7, 337 (1968)

2. Streater, R.: Commun. math. Phys. 7, 93 (1968)

3. Ruelle, D.: Statistical Mechanics. New York: Benjamin 1969

4. Takesaki, M.: Lecture Notes in Mathematics, No. 128. Berlin-Heidelberg-New York: Springer 1970

5. Ruskai, M. B.: Commun. math. Phys. 20, 195 (1971)

6. Ruelle, D.: Helv. Phys. Acta 45, 215 (1972)

7. Sirigue, M., Winnink, M.: Marseille preprint (1970) unpublished. See Winnink, M.: Statistical Mechanics and Field Theory (eds. R. Sen, C. Weil). Jerusalem: Keter Publishing House 1971 
8. Pulvirenti, M., Tirozzi, B.: Commun. math. Phys. 30, 83 (1973)

9. Duneau, M., Souillard, B.: Commun. math. Phys. 31, 113 (1973)

10. Haag, R., Hugenholtz, N., Winnink, M.: Commun. math. Phys. 5, 215 (1967)

11. Riesz,F., Nagy, R.: Lecon d'Analyse Fonctionelle. Paris: Gauthiers Villars (1965)

12. Wigner, E. P., Yanase, M.-M.: Proc. Nat. Acad. Sci. USA 49, 910 (1963)

13. Bratteli, O.: Comm. Pure Appl. Math. 25, 759 (1972)

14. Ginibre, J.: Les Houches Summer School (1970). New York: Gordon and Breach (1972)

15. Robinson, D. W.: Commun. math. Phys. 19, 219 (1970)

16. Ruelle, D.: Commun. math. Phys. 18, 127 (1970)

17. Suhov, Ju. M.: Unpublished manuscript

18. Courbage, M., Miracle-Sole, S., Robinson, D. W.: Ann. Inst. Henri. Poincaré 16, 71 (1971)

19. Novikov, I. D.: Funct. Anal. Appl. 4, $\star \star \star ~(1970)$

20. Suhov, Ju. M.: Russ. Math. Rev. 83, 491 (1970)

Communicated by J. L. Lebowitz

Received January 27, 1976 